\title{
Mechanical Properties of Single Motor Molecules Studied by Three-Dimensional Thermal Force Probing in Optical Tweezers
}

\author{
Sylvia Jeney, ${ }^{[\mathrm{b}]}$ Ernst H. K. Stelzer, ${ }^{[\mathrm{c}]}$ Helmut Grubmüller, ${ }^{[\mathrm{d}]}$ and Ernst- \\ Ludwig Florin*[a]
}

A new method combining three-dimensional (3D) force measurements in an optical trap with the analysis of thermally induced (Brownian) position fluctuations of a trapped probe was used to investigate the mechanical properties of a single molecule, the molecular motor kinesin. One kinesin molecule attached to the probe was bound in a rigorlike state to one microtubule. The optical trap was kept weak to measure the thermal forces acting on the probe, which were mainly counterbalanced by the kinesin tether. The stiffness of kinesin during stretching and compression with respect to its backbone axis were measured. Our results indicate that a section of kinesin close to the motor domain is the dominating element in the flexibility of the motor structure. The experiments demonstrate the power of $3 D$ thermal fluctuation analysis to characterize mechanical properties of individual motor proteins and indicate its usefulness to study single molecule in general.

\section{Introduction}

Micro- and nanomechanical properties can be studied by applying a force to an object and measuring its response. Nowadays, such force measurements are well-established even for a single molecule. Biopolymers, such as DNA and proteins, are investigated using force transducers such as optical traps, ${ }^{[1,2]}$ glass needles, ${ }^{[3]}$ atomic force microscopes ${ }^{[4,5]}$ or bio-force probes. ${ }^{[6]}$ Until now, single-molecule force measurements were mostly restricted to one dimension, neglecting the three-dimensional (3D) nature of mechanical properties of molecules. Recently, Block et al. extended the optical force clamp technique to two dimensions, ${ }^{[7]}$ which allowed new insights in the mechanochemical reaction cycle of kinesin, a molecular motor. ${ }^{[8]}$ This result demonstrates the importance of extending studies of single-molecule mechanics into further dimensions.

Herein, we present a method based on optical trapping to study time-resolved single-molecule mechanics not only in two but also in three dimensions. Our optical trap was equipped with a 3D position detector (Figure 1) to follow the motion of the trapped particle with a spatial precision in the nanometer range and a temporal resolution of a few tens of microseconds. ${ }^{[9]}$ Unlike in experiments with an optical force clamp, ${ }^{[8,10]}$ where high loads are used to reduce Brownian motion, we kept the optical forces to a minimum. This means that the applied forces are at least three orders of magnitude lower than in typical atomic force microscopy experiments, in the femtoNewton range. In this regime, thermal fluctuations of the molecule and the force transducer become significant and dominate the measurements. By developing an analysis method founded on Boltzmann statistics, we were able to extract mechanical and structural information from the 3D Brownian motion of the molecule under investigation and characterize its elastic response when exposed to mainly thermal forces. The understanding of the effects of such thermal forces, which are ubiquitous in biological systems, allows the performance of experiments in more physiological conditions.

To validate our method, we studied the behavior of a single kinesin molecule, while attached to its cytoskeletal counterpart, the microtubule. Kinesin is a molecular motor capable of converting the chemical energy gained by the hydrolysis of adenosine triphosphate (ATP) into mechanical work, thereby moving cargo along the microtubule track and ensuring intracellular transport. The structure and aspects of the mechanical function of kinesin have been intensively characterized ${ }^{[11,12]}$ and provide a solid base for the introduction of our new technique. Electron microscopic, biochemical, and other data suggest that kinesin consists of the following mechanical subunits: $:^{[13-18]}$ i) two enzymatic motor heads, which interact with the microtubule, and feature ii) a so-called neck-linker and a coiled-coil neck region, ${ }^{[19]}$ iii) a more than $50 \mathrm{~nm}$ long stalk, consisting of coiled-coil regions, assumed to be stiff with a pu-

[a] Prof. E.-L. Florin

Physics Department and Center for Nonlinear Dynamics

The University of Texas at Austin, 1 University Station, C1600

Austin, TX 78712-0264 (USA)

Fax: (+1)512-471-1558

E-mail:florin@chaos.utexas.edu

[b] Dr. S. Jeney

Institut de Physique de la Matière Complexe

Faculté des Sciences de Base, École Polytechnique Fédérale de Lausanne 1015 Lausanne (Switzerland)

[c] Dr. E. H. K. Stelzer

Cell Biology and Biophysics Program

European Molecular Biology Laboratory

Meyerhofstrasse 1, Postfach 10.2209

69117 Heidelberg (Germany)

[d] Prof. H. Grubmüller

Max-Planck-Institut für biophysikalische Chemie

Am Faßberg 11, 37077 Göttingen (Germany) 


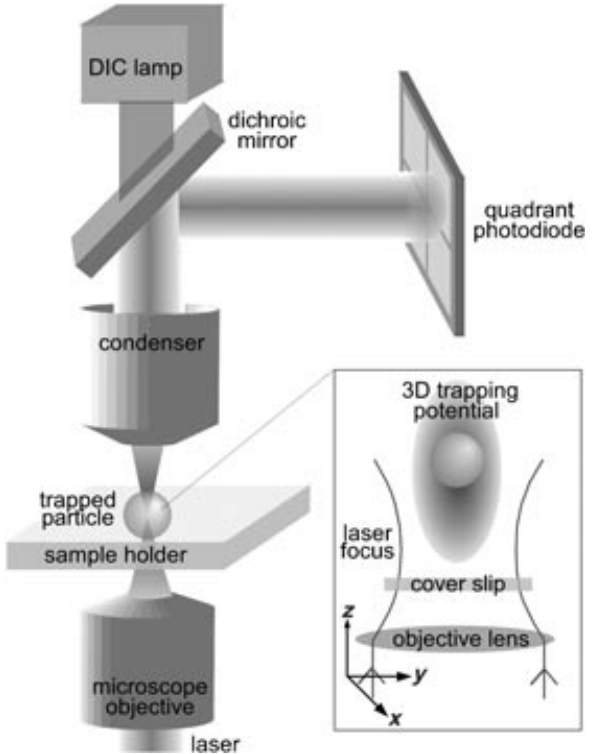

Figure 1. Scheme of the optical tweezers set-up. For calibration purposes a 430-nm diameter silica bead was held $2 \mu \mathrm{m}$ above the cover glass by the optical tweezers and its position fluctuations were recorded in the back focal plane onto a quadrant photodiode. Inset: Representation of the 3D optical trapping potential close to the laser focus.

tative flexible domain in the middle, and iv) two light chains at the end of the stalk where the cargo is bound.

The enzymatic activity implies several conformational state changes in the motor head domain, ${ }^{[20,21]}$ which are translated into displacements of the entire molecule several nanometers along the microtubule. To convert such structural changes into movement, the mechanochemical cycle must involve an amplification mechanism. In kinesin this seems to be achieved by a transition from a disordered to an ordered state of the neck linker sequence. ${ }^{[22,23]}$

For our study, we used a full-length drosophila kinesin mutant with one head missing. ${ }^{[24,25]}$ The system was simplified by choosing this single-headed mutant to avoid possible influences of the second head on our measurements. Since we were interested in the mechanical properties of the various mechanical elements of kinesin, we studied a nucleotide binding state in which kinesin is bound permanently to the microtubule. This can be achieved using the nonhydrolyzable ATP analogue AMP-PNP (adenosine $5^{\prime}$-( $\beta \gamma$-imido)triphosphate tetralithium salt hydrate). $430-\mathrm{nm}$ glass beads were functionalized with a stoichiometric ratio of one kinesin molecule per bead and attached to a microtubule by manipulation with optical tweezers. The thermal position fluctuations of the bead tethered to the microtubule by the single-headed kinesin construct were recorded on a 3D position detector. Using Boltzmann statistics, 3D energy landscapes were calculated from the probability distribution of the $3 \mathrm{D}$ position fluctuations. ${ }^{[26]}$

\section{Results and Discussion}

In this section we first introduce the technique of thermal force probing and show how it is used to calibrate the 3D po- tential profile of our laser trap. After calibration, the trapping potential served as a reference to compare with the potential profile resulting from additional tethering of the trapped bead by a single kinesin molecule. This procedure gives information on the length and mechanics of the kinesin molecule. The superposition of optical trapping and kinesin-tethering acting on the bead probe, as well as the effect of thermal and surface forces are discussed. Finally, the mechanical part along the kinesin structure mainly responsible for the measured elastic compliance between the trap and the motor is identified.

\section{D Thermal Force Probing and 3D Free-Energy Landscape of the Optical Trap}

A scheme of our experimental set-up is shown in Figure 1 and further details are given in the Experimental Section below and in ref. [27]. Typical 3D time-series of the thermal position fluctuations of a $430-\mathrm{nm}$ bead, trapped in the laser focus at $\approx$ $2 \mu \mathrm{m}$ above the cover-slip surface of the microscope sample chamber, were recorded on a quadrant photodiode and are shown in Figure 2a. Details on the 3D detection mechanism have been published in ref. [9]. During the observation time the particle moves, driven by thermal energy, inside a volume limited by the trapping potential well and the bead's interaction potential with its environment (as shown schematically in
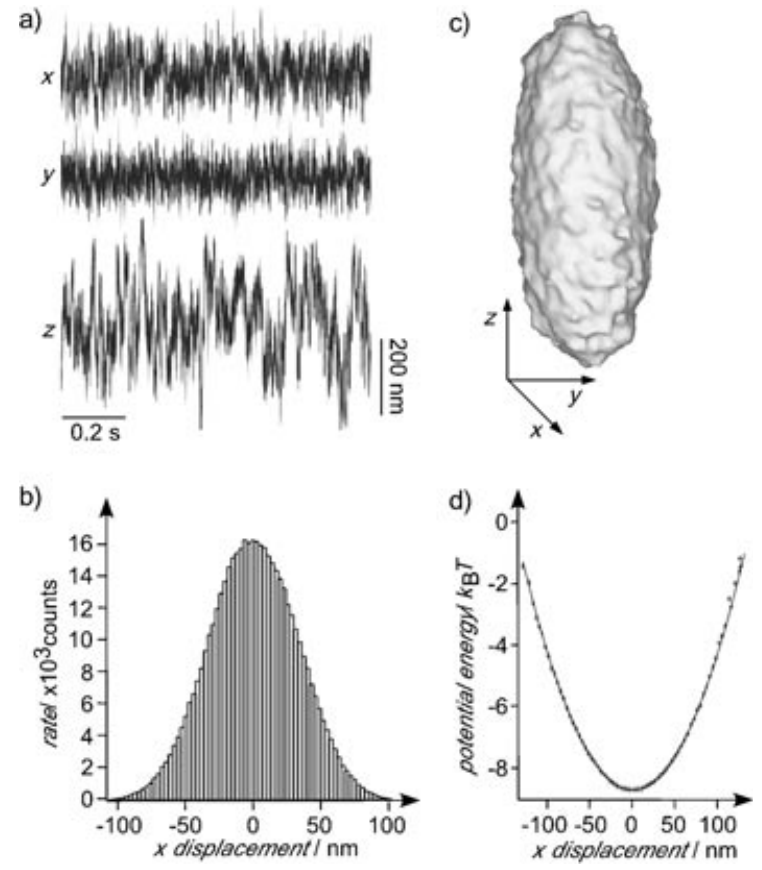

Figure 2. Determination of the 3D energy landscape of an optically trapped bead. a) 3D position fluctuation recordings of the bead at low laser power. b) The corresponding $1 D$ probability distribution of the lateral positions $\mathrm{x}(\mathrm{t})$ of the trapped bead. c) $3 D$ energy isosurface $\left(E_{i} \approx 5 k_{B} T\right.$ above the global energy minimum) of the trapping potential calculated from 3D position histograms using the data shown in $a$. The roughness of the surface is due to low counting statistics at higher energies and disappears as $\mathrm{E}_{\mathrm{i}}$ decreases. d) The lateral potential energy is given in units of $\mathrm{k}_{B} \mathrm{~T}$ and the zero position is chosen arbitrarily at the potential minimum. A parabolic curve (continuous line) fits very well to the data, which indicates the harmonicity of the potential. 
the close-up view of Figure 1). At a thermal equilibrium, the probability of finding a small particle in a given potential $V(\mathbf{q})$ inside a given volume dq follows the Boltzmann distribution [Eq. (1)]:

$\mathrm{P}(\mathbf{q}) \mathrm{d} \mathbf{q}=\frac{1}{C} \exp \left(-\frac{\mathrm{V}(\mathbf{q})}{k_{\mathrm{B}} T}\right) \mathrm{d} \mathbf{q}$

where $\mathbf{q}=(x, y, z)$ is the position vector of the particle, $C$ obeys the normalization condition $\int P(\mathbf{q}) \mathrm{d} \mathbf{q}=1, k_{\mathrm{B}}$ is the Boltzmann constant and $T$ is the temperature. All experiments were performed at room temperature $\left(\approx 27^{\circ} \mathrm{C}\right.$, for details $\left.s e e^{[27]}\right)$. Equation (1) states that the probability density of finding the bead at a certain position is directly correlated to the potential. Therefore, measuring the thermal position fluctuations of a particle can be used to calculate the $3 D$ potential $V(\mathbf{q})$ by Equation (2):

$V(\mathbf{q})=-k_{\mathrm{B}} T \ln P(\mathbf{q})+k_{\mathrm{B}} T \ln C$

The last term normalizes the probability distribution and results in an energy offset.

The probability density $P(\mathbf{q})$ of finding the trapped bead at position $q$ can be represented by a 3D histogram of the bead's possible positions inside the trap. After correction of the raw data for slow drifts of the laser intensity, the 3D position fluctuation histograms were calculated from 500000 position vectors. The bin-width of the histograms was chosen to strike a balance between spatial resolution and counting statistics. The overall shape of the histogram did not vary on changing its bin-width between 1 and 10 nanometers. Figure $2 \mathrm{~b}$ shows such a position histogram in one-dimension along the lateral $x$ direction of the laser trap. The 3D probability density distribution $P(\mathbf{q})$ can be then transformed into a 3D energy landscape using Equation (2). To display the resulting 3D free energy landscape $V(\mathbf{q})$ graphically, we calculated an energy isosurface at $\approx 5 k_{\mathrm{B}} T$, as shown in Figure $2 \mathrm{c}$. During the data acquisition time (usually $20 \mathrm{~s}$ ) more than $90 \%$ of all bead center positions were within the volume defined by this isosurface. Corresponding force and stiffness values were then calculated for each dimension from the first and respectively second derivative of the potential profile $V(\mathbf{q}) \cdot{ }^{[28]}$ All the potential profiles presented in the following were processed in the same way.

The dataset shown in Figure 2 is a typical calibration measurement of the optical trapping potential. The free energy isosurface appears elongated along the optical axis and has rotational symmetry in $x$ and $y$ (Figure $2 c$ ). This is expected from the intensity distribution in the focal spot of a high numerical aperture $(N A=1.3)$ objective lens. The elongation is due to a weaker force constant along the $z$ axis, which allows larger bead fluctuations than in the lateral directions. The exact axis ratio also depends on a number of parameters, for example, the bead properties and the shape of the laser focus. ${ }^{[29]}$ This $3 \mathrm{D}$ information could be then used to analyze the trapping potential along defined axes. In the case of the bead trapped in the potential of laser tweezers, these axes were chosen to be along and perpendicular to the optical beam axis. From the calibrated time-series in each dimension $x, y$ or $z$ (Figure $2 \mathrm{a}$ ), the 1D probability distribution of the particle's position was determined and plotted in Figure $2 b$ (shown only for $x(t)$ ). From this, a potential profile could be derived by using Equation (2; Figure $2 \mathrm{~d}$ ). The 1D position histogram shows a Gaussian distribution. The corresponding potential obtained is harmonic [Equation (3)] and confirms that for small displacements optical tweezers can in general be considered to act like a harmonic spring on the particle with a direction-dependent spring constant $k_{q}(q=x, y$ or $z)$ :

$V(x, y, z)=\frac{1}{2} k_{x} x^{2}+\frac{1}{2} k_{y} y^{2}+\frac{1}{2} k_{z} z^{2}$

The energy profile has the highest precision around its minimum, since the energy minimum is at the same time the most probable bead position and the most populated region in the histogram. The spring constant $k_{q}$ is given by the curvature (second derivative) of $V(\mathbf{q})$, which can be fitted by a parabolic function.

The 1D energy profiles along each axis could be fitted to harmonic potentials for displacements of up to several hundred nanometers (Figure $2 \mathrm{~d}$ ). The trapping force constants obtained were $k_{x}=2, k_{y}=3$, and $k_{z}=0.4 \mathrm{fN} \mathrm{nm}^{-1}$ and these provided the $3 \mathrm{D}$ force calibration of the optical trap.

Since no assumption about the shape of the potential was made, the method also applies to nonharmonic potentials. As is shown next, this method can be used for force measurements in $3 \mathrm{D}$ and in addition, it provides access to $3 \mathrm{D}$ energy profiles, regardless of their shape, with a resolution of $1 / 10$ of $k_{\mathrm{B}} T$ and sub-nanometer spatial resolution.

\section{D Free-Energy Landscapes of the Laser Trap plus the Kine- sin Tether}

To analyze the behavior of a bead tethered to a microtubule (MT) by kinesin, the lower cover slip of the microscope sample chamber was coated with dispersed MTs and the chamber was filled with a buffer solution containing kinesin-coated beads (silica, $430 \mathrm{~nm}$ in diameter) and casein $\left(1 \mathrm{mg} \mathrm{mL}^{-1}\right)$, which is known to reduce nonspecific interactions. MT orientation was verified by differential interference contrast (DIC) microscopy. After optical trap calibration, either a single bead, functionalized with single-headed kinesin, was moved close to the surface to allow interaction with the MT, or the laser trap was positioned onto a bead already attached to an MT (Figure 3). The carefully adjusted motor-to-bead ratio ensured that, on average, less than one kinesin molecule at a time was able to link the bead to the microtubule. ${ }^{[30]}$ Even after a 30 min sedimentation time, no bead was found to bind to the casein-passivated cover-slip surfaces, unless a MT was lying underneath the bead. The kinesin was bound specifically in the presence of $2 \mathrm{~mm}$ AMP-PNP.

In the experiment shown in Figure 3 a the optical trap was displaced laterally and axially towards the MT, which caused an average pulling force $\mathbf{F}_{\text {trap }}$ on the molecular tether of $\left(F_{x} \approx\right.$ $-300, F_{y} \approx-130, F_{z} \approx-50 \mathrm{fN}$ ) with the axial force component 
a)
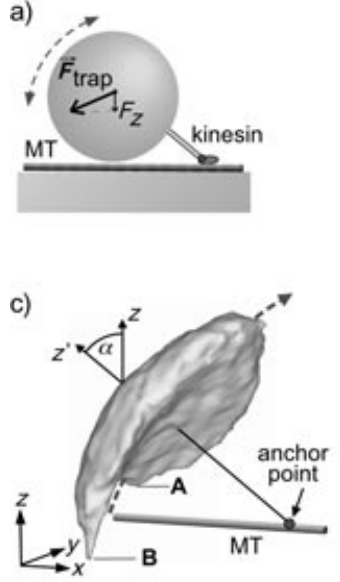

e)

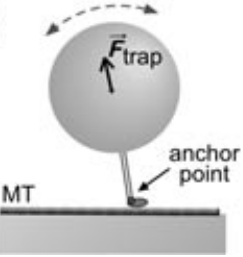

b)

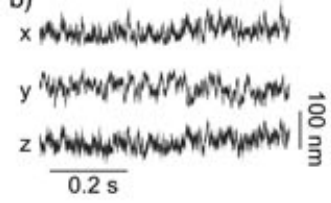

d)

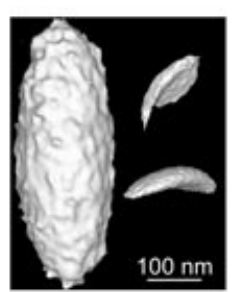

f)

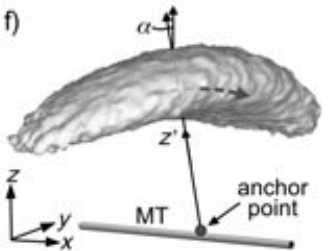

Figure 3. 3D free-energy landscapes of a tethered bead. a) A bead tethered to a cover-slip-adsorbed MT by an individual single-headed kinesin molecule was trapped in the presence of $2 \mathrm{mM}$ AMP-PNP. A force $\mathbf{F}_{\text {trap }}$ small enough to allow large thermal position fluctuations of the bead, was applied by displacing the optical trap laterally and axially. The axial component $\mathrm{F}_{\mathrm{z}}$ of the trapping force pointed towards the cover glass. Thermal fluctuations allowed the bead to fluctuate around the anchor point in all directions. b) Typical fluctuation recordings for the tethered bead. c) Free energy isosurface calculated from the data of the tethered bead showing a shape similar to a section of a spherical shell. $A$ and $B$ denote the upper and the lower levels of the step along the y axis. The black diagonal line indicates the average orientation of the energy landscape and thus of the linker axis relative to the MT axis and to the optical (z) axis. The distance of the free energy landscape to the MT is not drawn to scale. $\alpha$ denotes the average tilt angle of the energy landscape in the global potential minimum relative to the optical axis. The minimum was located along the linker axis in the center of the $3 D$ energy profile. d) For comparison, both presented energy isosurfaces of beads tethered by a single kinesin molecule to the MT are drawn to scale. e) In a different experiment the external force $\mathbf{F}_{\text {trap }}$ pointed away from the glass surface. $d$ ) Corresponding free energy landscape.

$F_{z}$ pointing towards the glass surface. The corresponding average displacement of the bead center from the optical trap center was $\Delta x=164, \Delta y=43$, and $\Delta z=130 \mathrm{~nm}$. The attachment of the bead to the MT through the single kinesin molecule drastically restricted the bead diffusion inside the laser trap. Compared to a nontethered, trapped bead (Figure 2a), the amplitudes of the position fluctuations were significantly reduced in all three directions $x, y$, and $z$ and now had similar magnitudes (Figure $3 \mathrm{~b}$ ). The corresponding 3D free-energy profile had a different shape, similar to a piece of spherical shell that is tilted towards the MT and cover-slip surface. (Figure $3 c, d$ ). The position fluctuation amplitudes as measured in 1D (Figure $3 \mathrm{~b}$ ) appear symmetric in all three dimensions, which gives a distorted view of the real $3 D$ data. This again demonstrates the information obtained by the 3D analysis of optical trapping experiments. In Figure $3 \mathrm{e}$ a similar experiment is presented, where the pulling force $\boldsymbol{F}_{\text {trap }}$ was smaller and

pointed away from the surface $\left(F_{x}=65, F_{y}=93\right.$, and $\left.F_{z}=76 \mathrm{fN}\right)$. The 3D energy profile (Figure $3 \mathrm{f}$ ) also resembles a part of a spherical shell with a similar size. Figure $3 \mathrm{~d}$ shows a drawn-toscale comparison of the 3D free-energy profiles of the trapping potential and of the two tethering potentials in the presented experiments, and points out that the rotational symmetry of the trapping potential around the optical axis vanished completely. The strong restriction of the bead fluctuations after tethering gives first indications that the kinesin molecule behaves like a relatively "stiff" rod, connecting the MT and the bead.

It should be kept in mind that the shape of the 3D free energy landscapes as represented in Figure $3 \mathrm{~d}$ and the shape of the corresponding 3D position histogram are equivalent, since they are related to each other by Equation (2).

\section{Kinesin Orientation and Length}

One outstanding feature of all the free energy landscapes-position histograms measured in $N=100$ experiments is their spatial orientation (see Figure 3 ). This observation agrees well with the assumption that the kinesin stalk acts as a linker between the MT and bead, pivoting around its anchor point. Surprisingly, the linker does not behave like a random polymer chain, ${ }^{[31]}$ but develops some restoring forces against thermal forces. In most cases, it appears to be tilted at an angle $\alpha$ relative to the optical axis. To determine the orientation of the kinesin stalk, we reduced the $3 D$ profile to a $2 D$ "free-energy minimum surface" by calculating the energy minima of $1 D$ free-energy profiles at each position in the $x-y$ plane along the $z$ axis (optical axis of the trap). The free energy minimum surface shown in Figure 4 is calculated from the 3D free energy profile presented in Figure $3 \mathrm{c}$. In all the experiments performed $(N=100)$, we found only one absolute minimum within the free-energy landscape probed. An average orientation of surface elements around the global energy minimum

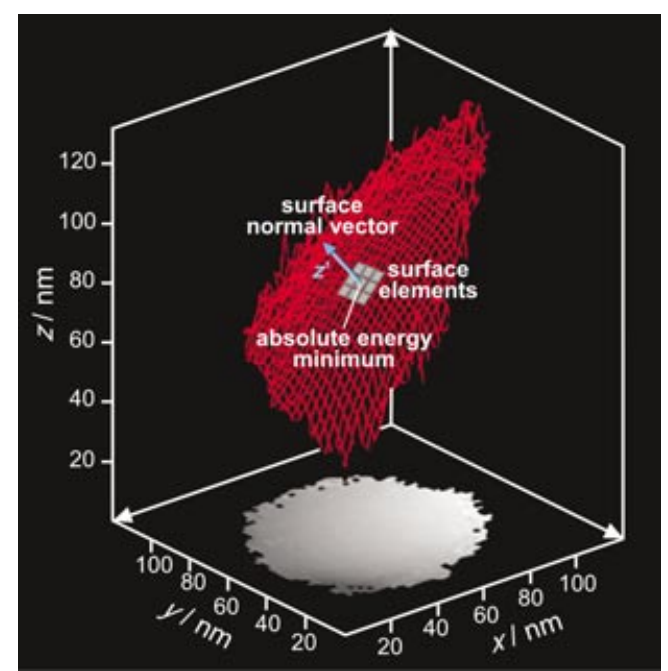

Figure 4. Free energy minimum surface calculated from the $3 D$ free energy profile presented in Figure $3 \mathrm{c}$. The surface normal vector gives the orientation of the molecular tether and has its origin in the absolute free-energy minimum. 
was then calculated. The resulting surface normal vector points in the direction of the average orientation of the energy profile. We postulate that the overall orientation of the 3D position histogram corresponds to the orientation of the linker molecule when slightly stretched. Therefore, we can align the center of the bead, the tether axis, and the anchor point of the kinesin head on the MT onto a common axis $z^{\prime}$ (Figure $3 c, f$ ), which is independent of the optical axis given by our quadrant photodiode. Hence this newly defined $z^{\prime}$ axis is parallel to the kinesin stalk and its origin corresponds to the point of highest probability for the bead's center positions: the overall minimum of the 3D potential (Figure 4).

From the orientation of the surface normal vector, average tilt angles $\alpha$ of $\approx 43^{\circ}$ and $\approx 9^{\circ}$ were determined for the profiles shown in Figures $3 \mathrm{c}$ and $3 \mathrm{f}$, respectively. The maximal possible tilt angle $\alpha_{\max }$ of the energy landscape was reached in the experiment in Figure $3 c$, and the tethered bead occasionally touched the cover slip or MT surface which lay underneath. $\alpha_{\max }$ is determined by the linker length $/$ and the bead radius $r$ as shown in Equation (4):

$\alpha_{\max }=\arccos \frac{r}{r+1}$

For the maximum tilt angle of $43^{\circ}$ and bead-radius of $215 \mathrm{~nm}$ with Equation (4) we find a linker length of $\approx 78 \pm 6 \mathrm{~nm}$. This length matches well with electron microscopy data for a fulllength kinesin molecule. ${ }^{[32]}$ As a control we performed the same experiments with larger beads $(900 \mathrm{~nm}$ in diameter). They showed, as expected, smaller maximum tilt angles, but the data evaluation resulted in the same linker length.

The "step" between level A and B in the 3D position histogram depicted in Figure $3 \mathrm{C}$ can be interpreted as the bead being either in contact with the MT (position A) or with the glass surface (position B). The height difference of $22 \pm 3 \mathrm{~nm}$ between points $A$ and $B$ agrees well with the thickness of one MT, which is known to be $25 \mathrm{~nm}$. In fact, 3D position histograms represent the volume scanned by the bead only driven by thermal forces. If the bead is placed by manipulation with optical tweezers close to an obstacle (in this case it was an MT), its presence will force the bead to avoid the space occupied by the MT, thereby rendering a topographical image of it. This idea and concept of "thermal noise imaging" was demonstrated by Tischer et al. ${ }^{[33]}$

Interpretation of our data relies on the assumption that kinesin is attached in the right orientation, that is, with its tail to the probe. The bead-coating method described in the experimental section was reliable for recombinant proteins as long as the tail of the kinesin heavy chain was present in the purified molecule. Beads coated with kinesin mutants that were missing this tail only showed low affinity to MT unless they were attached to the bead by an antibody at the extremity of the construct (data not shown). Furthermore, it is known from classical kinesin/MT-gliding assays, ${ }^{[34]}$ that the presence of casein in the buffer favors the correct orientation of kinesin on a glass surface, leaving the head domain free to interact with an MT.

\section{Considerations on the Trapping, Tethering, Thermal, and Surface Forces Involved}

The volume the bead can probe, when only driven by thermal forces, is limited by the combined effect of two potentials: the laser trapping potential $V_{\text {trap }}$ and the potential generated by the mechanical properties of the tethering molecule $V_{\text {tether }}$ In the absence of any additional laser-trapping potential and for a simple polymeric tether, the bead should explore a surface volume like a segment of a sphere, limited on its edges only by the interaction of the bead with the surface it is bound to. An additional laser-trapping potential restricts the accessible volume depending on the relative strength of $V_{\text {trap }}$ and $V_{\text {tether }}$ as well as on their relative position. As measured, the resulting potential $V_{\text {tot }}$ has a single minimum, which is shifted according to the position and strength of its components $V_{\text {trap }}$ and $V_{\text {tether }}$ Consequently, the application of a load tilts the free energy landscape along the direction of loading. This effect can be clearly seen in the second experiment shown in Figure $3 \mathrm{e}, \mathrm{f}$. Here, a load $\left(\boldsymbol{F}_{\text {trap }}\right)$ was applied to the bead that pointed away from the cover-glass surface and the MT. Therefore, the part of the mechanical potential pointing along the optical axis was probed.

If $V_{\text {trap }}$ and $V_{\text {tether }}$ are both harmonic, a relative displacement $x_{0}$ between the two potentials should not lead to a change in the shape of the energy landscape, because the curvature of the resulting energy landscape is only given by the sum of the two force constants and does not change with the relative position of the potentials to each other, as shown by Equation (5):

$$
\begin{aligned}
& V_{\text {tot }}=\frac{1}{2} k_{\text {tether }} x^{2}+\frac{1}{2} k_{\text {trap }}\left(x+x_{0}\right)^{2}=\frac{1}{2}\left(k_{\text {tether }}+k_{\text {trap }}\right) x^{2} \\
& +k_{\text {trap }} x x_{0}+\frac{1}{2} k_{\text {trap }} x_{0}^{2}
\end{aligned}
$$

The linear term $k_{\text {trap }} X x_{0}$ allows a trapped particle to reach a part of the potential landscape shifted away from its minimum but does not alter the shape of the landscape. The constant term provides only an energy offset. In practice, the shift $x_{0}$ occurs when the axis of the laser trap potential does not match exactly with the one of the molecule-to-molecule linkage.

To probe the elastic properties of the kinesin tether, we minimized the trapping force constant $k_{\text {trap }}$. The elastic response of kinesin to thermal forces became dominant over the trapping force and contributed almost exclusively to the shape of the free energy landscape.

Within the precision of our technique, we found no significant influence from van der Waals or electrostatic forces arising from the cover-glass surface proximity to the measured potential profiles. We performed most experiments with the bead held at least $40 \mathrm{~nm}$ away from the MT surface (see, for example, Figure $3 \mathrm{e}$ ). At this distance, surface forces should have been negligible. 


\section{Stiffness of Kinesin along its Linker Axis}

As presented in the following $V_{\text {tot, }}$ and hence $V_{\text {tether }}$ was not harmonic, which rules out the possibility that kinesin behaves as a simple linear spring. To investigate the mechanical properties of kinesin along its longitudinal axis, 1D profiles of the free energy, force, and stiffness versus extension (Figure 5) were calculated from the 3D energy landscape in Figure $3 \mathrm{C}$ along the linker axis $z^{\prime}$ and going through the potential minimum. To increase the precision of the energy profiles, we averaged neighboring profiles $(\mathrm{N} \approx 5)$ along parallel paths, separated from each other by approximately the bin width (several nanometers) of the position histograms. The free-energy profile (Figure 5) had the highest precision of $\approx 0.1 k_{\mathrm{B}} T$ around its minimum, which is the most populated position in the histogram. The errors in the $1 \mathrm{D}$ energy profile are defined as the standard deviation of the different energy values $(\mathrm{N} \approx 5)$ used for the averaging process. In the timeframe of our measurements only $\approx 8 \mathrm{k}_{\mathrm{B}} \mathrm{T}$ were explored, hence the number of points per bin drops to zero at higher energy levels. The contribution of the optical trapping potential to the 1D energy profile along the linker axis was small in all our experiments, because its stiffness was at least two orders of magnitude smaller than the maximal stiffness of the linker.

The energy profile shown in Figure 5 is asymmetric with the shallower part pointing towards the anchor point, that is, the kinesin head. The corresponding force profile (Figure 5, force) was calculated from the first derivative of the energy profile.

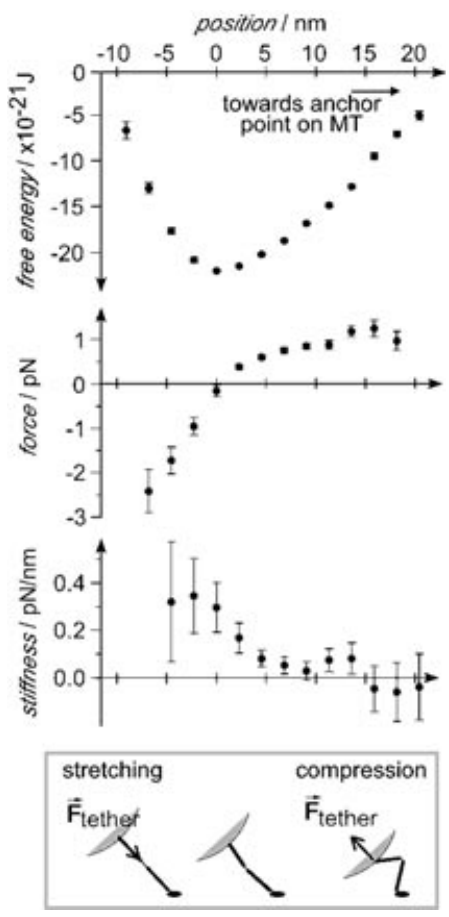

Figure 5. Free energy, force, and stiffness profiles along the linker axis through the global minimum of the 3D energy landscape shown in Figure $3 c$. Only the part around the potential minimum is shown. The bottom panel illustrates possible conformations and behaviors of the kinesin for different parts of the energy landscape. The left side corresponds to a stretching and the right side to a compression of the kinesin.
These forces ranged from -2.5 to $1 \mathrm{pN}$. It should be kept in mind that these are forces that kinesin generates to react to the action of the random external thermal forces acting on the longitudinal axis of the kinesin-bead system. The forces exceeded the external force applied by the optical trap by at least one order of magnitude. In the case of negative forces, kinesin withstands stretching, and in the case of the positive forces, it withstands compression. The stiffness profile (Figure 5) was calculated from the second derivative of the free-energy profile. In fact, two stiffness regimes are observable. The kinesin tether has a relatively high stiffness to stretching of $\approx 0.3 \mathrm{pN} \mathrm{nm}^{-1}$ and a lower stiffness to compression of $\approx 0.05 \mathrm{pNnm}^{-1}$. These values varied from experiment to experiment, depending on parameters such as the direction and value of the applied trapping force, but for all $N=100$ experiments they were on the same order of magnitude. A very surprising result is that kinesin not only generated an absolute restoring force of up to $2.5 \mathrm{pN}$ to stretching, but also a restoring force of up to $1 \mathrm{pN}$ to compression (Figure 5). The force limits in Figure 5 are given by the limited position statistics in this particular experiment. Although this experiment is technically very challenging, one can measure a large number of points and thus explore the entire energy landscape until the kinesin unbinds from the MT and the bead diffuses away.

The asymmetric potential shown in Figure 5 resembles a typical potential expected for a semiflexible polymer, where the steeply increasing part is a result of the stretching of the filament and the shallower part is typical for Euler buckling under compressive forces. ${ }^{[35-37]}$ However, the system we are studying consists of several elastic elements, and the kinesin tether itself is known to be inhomogeneous, with mechanically complex subunits. Each of these parts will be discussed in the following, to identify which one is more likely to be responsible for the measured elastic response.

\section{Origin of the Measured Elastic Response: The Most Flexible Element in the Kinesin Structure}

In principle, the elastic response is given by the entire MT-kinesin bead system, including the linkage of kinesin to the bead and the linkage of the MT to the cover-slip surface. Each of these elastic elements acts in series, with the softest element dominating the overall elastic response. The coupling between MTs and glass was shown to be very strong in our own AFM studies using a similar preparation (unpublished results). Therefore, we do not expect significant elastic contribution from this coupling. The coupling between kinesin and the bead is presumably also strong, because proteins attach to glass surfaces through short-range forces, leaving no room for a major elasticity. This view is supported by the observation of the repulsive potential along the linker axis, which suggests a stiff kinesin bead linkage. For these reasons, we attribute the measured elastic response to the kinesin molecule itself, taking into account that the optical trapping forces were negligible.

Figure 6 outlines the simplest and most common model of the mechanical elements along the full-length kinesin structure. All features are drawn to scale. The major part consists of 


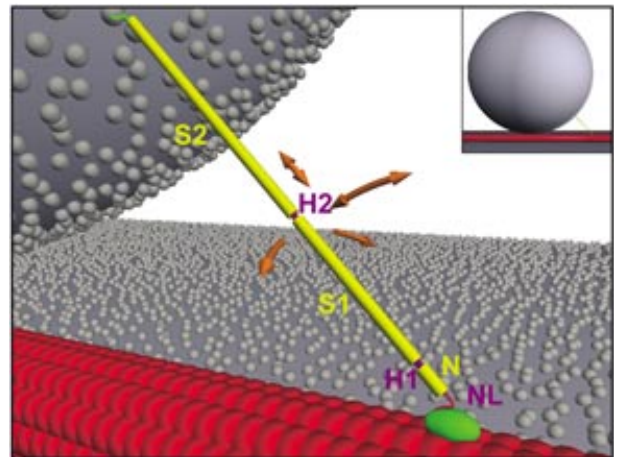

Figure 6. Illustration of the force experiment (drawn to scale) when the maximal tilt angle $\alpha_{\max }$ is reached. For simplicity the single-headed kinesin molecule consists of simple mechanical elements. $\alpha$-Helical coiled-coil structures (neck coiled-coil (N), stalk 1 (S1) and stalk 2 (S2) are considered as stiff rods (yellow cylinders), stiff globular objects (head and tails) are shown in green, and assumed flexible molecular joints are shown in purple (hinge $1(\mathrm{H} 1)$ and hinge 2 (H2)) and red (neck-linker NL). All other elements (cover slip (gray), microtubule (dark red), and bead) and their connections are assumed to be rigid. Casein molecules, added to reduce nonspecific interaction, are shown as light gray spheres on the bead and on the cover slip.

two $\alpha$-helical coiled-coil structures called stalk 1 (S1), stalk 2 (S2) and the $\alpha$-helical coiled-coil portion of the neck (N). ${ }^{[11,20]}$ These structures (Figure 6; yellow rods) are supposed to be stiffer than more disordered parts of kinesin, such as the neck linker. To confirm this, we performed molecular-force-dynamics simulations $s^{[38]}$ for the leucin zipper, a prototype for $\alpha$-helical coiled-coil structures. Using its X-ray structure, ${ }^{[39]}$ (Brookhaven Data Bank entry 2zta), an atomic model of the coiled-coil embedded in aqueous solution was constructed and subsequently equilibrated for $50 \mathrm{ps}$. The simulated system was elliptical $(86 \times 72 \times 71 \AA)$ and consisted of a total of 21656 atoms. All simulations were performed using the parallel computer program EGO VIII ${ }^{[40]}$ with full electrostatics. ${ }^{[41]}$ During the simulations, the protein was stretched by applying pulling forces parallel to the helix bundle on the nitrogen atoms of Gln 4 and Gln 35, and, in the opposite direction, on the (backbone carbonyl) carbon atoms of Leu 26 and Leu 57. Accordingly, two monoharmonic pulling potentials (for definition, see ref. [38]) with a spring constant of $7 \mathrm{pN} \AA^{-1}$ were moved with velocities between 0.5 and $5 \AA_{p s^{-1}}$ each. (Note that the termini of the helices moved much more slowly becuase of their small spring constant.) For velocities below $1 \AA \mathrm{s}^{-1}$ frictional forces turned out to be negligible. From the forces exerted and the resulting elongation of the helices, and assuming a cross section of $300 \AA^{2}$, a Young's modulus of between 2 and $5 \mathrm{GPa}$ was estimated. This value corresponds to a stiffness of $\approx 85 \mathrm{pN} \mathrm{nm}^{-1}$ for a 70-nm long coiled-coil structure and is two to three orders of magnitude higher than the maximum measured kinesin stiffness in our experiments. Therefore, we assume that S1 and $\mathrm{S} 2$ and $\mathrm{N}^{[42]}$ act in our experiment as stiff rods that can neither be bent, stretched, nor compressed significantly.

The globular structure of the motor domain is well-known and has been reported also to be stiff. ${ }^{[13]}$ In addition, the head domain is short in comparison to the total length of kinesin, hence its elastic deformation would contribute little to the total elastic fluctuations of the kinesin.
The remaining sources of flexibility that could contribute to the measured elastic response of kinesin are its molecular joints (Figure 6) between head and neck ("neck-linker" NL), between neck and stalk 1 (hinge $\mathrm{H} 1$ ), between stalk 1 and stalk 2 (hinge $\mathrm{H} 2$ ), and between $\mathrm{S} 2$ and the bead. All these regions could contribute, either through stretching or bending. As indicated in Figure 5 (bottom panel) these molecular joints may indeed contribute to the overall elastic response of kinesin at higher forces (Figure 5) and allow the probing particle to move more than $50 \mathrm{~nm}$ along the linker axis. However, only flexibility in a joint very close to the anchoring point on the MT would result in the pivoting of a tether as long as the contour length of kinesin, as measured here. Therefore, the softest elastic element in our system has to coincide with the region between $\mathrm{H} 1$ and the motor domain. This region has been shown to have strong influence on the velocity and directionality of kinesin. ${ }^{[43-45]}$ Chemical or genetic changes in or close to the motor domain should then have an influence on the measured 3D free-energy landscapes and unambiguously confirm our results. A comparative study with different motor constructs in different nucleotide-binding states will be performed.

\section{Conclusions}

Herein, we demonstrate that the extension of single molecule force experiments to $3 \mathrm{D}$ and into the thermal fluctuation regime provides unprecedented access to $3 \mathrm{D}$ molecular mechanics and allows a detailed characterization of the mechanical properties of an individual kinesin molecule. We show that the kinesin molecule is able to generate restoring forces against compression along its stalk. From our data, we can conclude that the neck region of kinesin is the dominating elastic element along the kinesin structure. The presented measurements were chosen from many sets $(N=100)$, but each individual energy profile measured depends on the trapping potential, the applied external force and the orientation of the force with respect to the MT-binding site. Therefore, each profile has to be evaluated on an individual basis, providing different views of the energy landscape of a molecule.

The molecular mechanics of kinesin is only one example of a detailed investigation of molecular mechanics in 3D and in the thermal energy regime. For the first time, molecular joints become accessible to direct mechanical measurements, providing new insights into their structure-function relation. Also, the monitoring of different conformational states over an extended period in a single molecule may become possible by measuring changes in its mechanical properties.

\section{Experimental Section}

A detailed description of all protocols used is given in ref. [27].

Microtubules were polymerized in BRB80 [80 mM piperazine- $N, N^{\prime}-$ bis(2-ethanesulfonic acid) (PIPES), $2 \mathrm{mM} \mathrm{MgCl}, 1 \mathrm{~mm}$ ethylene glycol-bis(2-aminoethylether)- $N, N, N^{\prime}, N^{\prime}$-tetraacetic acid (EGTA), pH 6.9] in the presence of $1 \mathrm{~mm}$ guanosine triphosphate (GTP) at $37^{\circ} \mathrm{C}$ for $30 \mathrm{~min}$ and fixed in BRB80 with $15-\mu \mathrm{M}$ taxol. The concen- 
tration of the microtubules was adjusted in taxol/BRB80 to give a sparse coating of the microscope chamber.

Kinesin mutants: We used a single-headed kinesin mutant expressed in bacteria as a full-length protein (kindly provided by W. O. Hancock, Penn State University, USA). This mutant lacks one of the two globular head domains but is still able to dimerize and has a coiled-coil stalk region followed by a tail. Multiple motor gliding assays were performed systematically before coating of beads with a new batch of proteins. In vitro motility assays of the same single-headed kinesin molecules used in our work are described in Hancock et al. ${ }^{[46]}$

Bead-coating with single motor molecules was achieved by modifying a standard protocol. ${ }^{[47]}$ Fluorescent silica beads (430 nm diameter, kindly provided by $A$. van Blaaderen, ${ }^{[48]}$ ) were preincubated for $5 \mathrm{~min}$ in assay buffer $\left(80 \mathrm{~mm}\right.$ PIPES, $4 \mathrm{~mm} \mathrm{MgCl}_{2}, 1 \mathrm{~mm}$ EGTA, $\mathrm{pH} 6.9,1 \mathrm{mg} \mathrm{mL}^{-1}$ filtered casein, $50 \mathrm{mM} \mathrm{KCl}, 1 \mathrm{~mm}$ dithiothreitol, $20 \mu \mathrm{M}$ taxol, $2 \mathrm{~mm}$ AMP-PNP), to precoat the beads with casein, and then incubated at $4{ }^{\circ} \mathrm{C}$ for at least 1 hour with a diluted kinesin solution. The molar kinesin-to-bead ratio was adjusted between 1 and 2. We used Poisson statistics as described by Block et al. ${ }^{[47]}$ and others to estimate the amount of active motor molecule per bead. Our experiments were performed with a binding probability $P(n)=$ 0.55 , which corresponded in our Poisson statistical analysis to a kinesin-to-bead ratio of 1 . The precoating of the beads with casein molecules saturates the surface and therefore avoids a clustering of several kinesin molecules onto only few beads, thereby ensuring a minimum of kinesins per bead.

Microscope chamber: The flow chamber consisted of two cover slips stuck to each side of a stainless steel holder and magnetically fixed to an inverted microscope stage. The cover slips $(22 \times 22 \mathrm{~mm}$, Clay Adams, USA) were previously washed by boiling them for $15 \mathrm{~min}$ in saturated $\mathrm{KOH}$-ethanol solution, followed by several rinses in absolute ethanol. To guarantee a tight binding of the microtubules to the cover slip, the glass surface was modified with 3trimethoxysilylpropyl-diethylenetriamine (Huls, Germany).

A diluted microtubule-solution was perfused into the microscope chamber at high flow rates, which ensured that they bound with their long axis parallel to the flow field. The microtubules were allowed to bind to the pretreated lower glass-slide for $10 \mathrm{~min}$. The chamber was then washed with assay buffer to remove unbound microtubules. This wash also coated the cover slip with casein, which minimized non-specific adhesion between the kinesincoated beads and the chamber surface. After 5 min incubation, the motor-coated beads were added.

Optical trap and 3D particle tracking: (Figure 1) Our optical trap (for more details see refs. $[27,49,50]$ ) is built around an inverted optical microscope capable of differential interference contrast (DIC) microscopy (Axiovert 35, Zeiss, Germany). An NIR laser, operating at a wavelength of $1064 \mathrm{~nm}$ (T20-B10-106Q, Spectra Physics, Germany), is coupled into the back aperture of an objective lens, as in a confocal microscope, and serves as an optical trap. To increase the mechanical stability of the instrument, the sample, the objective lens and the condenser lens are integrated into one mechanical unit (EMBL, Heidelberg, Germany). The forward scattered light from the bead trapped behind the laser focus is collimated by the condenser lens of the microscope and directed by a dichroic mirror onto a quadrant photodiode (S5981, Hamamatsu, Germany) located at the backfocal plane of the condenser lens. The dichroic mirror is placed between the condenser lens and the field aperture to separate the trapping laser light from the DIC illumination. To measure the 3D position of the bead in the optical trap, the differential signals from the quadrant photodiode and the total intensity scattered onto it are measured. The differential signals serve as the lateral position signals and the sum signal of all quadrants as the axial position signal. The electronic detection bandwidth is $\approx 50 \mathrm{kHz}$ in all three dimensions. All directions show a linear response and are independent from each other for small displacements (for details see ref. [49]). The electronic signals are fed through filter amplifiers (SA 5000, EMBL, Germany) to make full use of the 12-bit resolution of the data acquisition board (ADWin F5, Jäger Electronics, Germany). The fluctuations were recorded in three dimensions for typically $20 \mathrm{~s}$ at a sampling rate of $25 \mathrm{kHz}$.

Detector calibration: To calibrate the detector response for small displacements, a $430 \mathrm{~nm}$ diameter silica bead was trapped at a distance of $\approx 2 \mu \mathrm{m}$ from the glass surface (Figure $1 \mathrm{a}$ ). This distance ensures that hydrodynamic corrections, as well as changes in the force constant along the optical axis due to spherical aberration effects, are negligible. ${ }^{[50]}$ The power of the trapping laser was adjusted to allow thermally driven lateral position fluctuations (in $x$ and $y$ ) of up to $\pm 150 \mathrm{~nm}$ (Figure $1 \mathrm{~b}$ ). Under these conditions the laser power was well below $10 \mathrm{~mW}$ at the specimen plane. The sensitivity of the detector for all three dimensions was calibrated by calculating the plateau value of the power spectral density (PSD) multiplied by the square of the frequency in an interval between 3 and $10 \mathrm{kHz}$. The detector sensitivity $\beta_{q}$ in $\mathrm{Vm}^{-1}$ along the axis $q$ was then calculated from the plateau value $p_{q}$ using Equation (6):

$\beta_{q}=\sqrt{3 \pi 3 \eta p_{q} d / k_{\mathrm{B}} T}$ with $q=x, y, z$

where $\eta$ denotes the viscosity of the medium, $d$ the diameter of the sphere, and $k_{\mathrm{B}}$ the Boltzmann constant (for details see ref. [51]).

\section{Acknowledgements}

We thank Heinrich Hörber, Rolf Eckert, Arnd Pralle, Joe Howard, Martha Liley, Bharat Bhushan and Manfred Schliwa for helpful discussions and assistance. We thank Will Hancock (University of Washington, Seattle) for providing us with single-headed kinesin. This work was supported by the Deutsche Forschungsgemeinschaft (DFG).

Keywords: mechanical properties - molecular motors - optical tweezers $\cdot$ single-molecule studies $\cdot$ thermal fluctuations

[1] K. Svoboda, C. F. Schmidt, B. J. Schnapp, S. M. Block, Nature 1993, 365, 721.

[2] J. T. Finer, R. M. Simmons, J. A. Spudich, Nature 1994, 368, 113.

[3] A. Kishino, T. Yanagida, Nature 1988, 334, 74.

[4] E.-L. Florin, V. T. Moy, H. E. Gaub, Science 1994, 264, 415.

[5] M. Rief, M. Gautel, F. Oesterhelt, J. M. Fernandez, H. E. Gaub, Science 1997, 276, 1109.

[6] R. Merkel, P. Nassoy, A. Leung, K. Ritchie, E. Evans, Nature 1999, 397, 50.

[7] M. J. Lang, C. L. Asbury, J. W. Shaevitz, S. M. Block, Biophys. J. 2002, 83, 491.

[8] S. M. Block, C. L. Asbury, J. W. Shaevitz, M. J. Lang, Proc. Natl. Acad. Sci. USA 2003, 100, 2351.

[9] A. Pralle, M. Prummer, E. L. Florin, E. H. K. Stelzer, J. K. H. Horber, Microsc. Res. Tech. 1999, 44, 378.

[10] K. Visscher, M. J. Schnitzer, S. M. Block, Nature 1999, 400, 184.

[11] M. Schliwa, G. Woehlke, Nature 2001, 411, 424.

[12] R. D. Vale, R. Case, E. Sablin, C. Hart, R. Fletterick, Philos. Trans. R. Soc. London 2000, 355, 449.

[13] F. J. Kull, E. P. Sablin, R. Lau, R. J. Fletterick, R. D. Vale, Nature 1996, 380, 550. 
[14] R. D. Vale, J. Cell Biol. 1996, 135, 291.

[15] S. Sack, J. Muller, F. Kozielski, A. Marx, M. Thormahlen, V. Biou, A Thompson, E. M. Mandelkow, S. Brady, R. Mandelkow, Molecular Biology of the Cell 1997, 8, 1505.

[16] F. Kozielski, D. Svergun, G. Zaccai, R. H. Wade, M. H. J. Koch, J. Biol. Chem. 2001, 276, 1267.

[17] W. R. Schief, J. Howard, Curr. Opin. Cell Biol. 2001, 13, 19.

[18] N. Naber, T. J. Minehardt, S. Rice, X. R. Chen, J. Grammer, M. Matuska, R. D. Vale, P. A. Kollman, R. Car, R. G. Yount, R. Cooke, E. Pate, Science 2003, 300, 798.

[19] M. Thormahlen, A. Marx, S. Sack, E. Mandelkow, J. Struct. Biol. 1998, 122, 30.

[20] R. D. Vale, R. J. Fletterick, Annual Review of Cell and Developmental Biology 1997, 13, 745.

[21] S. Rice, A. W. Lin, D. Safer, C. L. Hart, N. Naber, B. O. Carragher, S. M. Cain, E. Pechatnikova, E. M. Wilson-Kubalek, M. Whittaker, E. Pate, R. Cooke, E. W. Taylor, R. A. Milligan, R. D. Vale, Nature 1999, 402, 778.

[22] R. D. Vale, R. A. Milligan, Science 2000, 288, 88.

[23] H. Sosa, E. J. G. Peterman, W. E. Moerner, L. S. B. Goldstein, Nat. Struct. Biol. 2001, 8, 540.

[24] W. O. Hancock, J. Howard, Molecular Biology of the Cell 1996, 7, 1243.

[25] W. O. Hancock, J. Howard, Biophys. J. 1997, 72, MP204.

[26] E.-L. Florin, A. Pralle, E. H. K. Stelzer, J. K. H. Hörber, App. Phys. A 1998, 66, S75.

[27] S. Jeney, E.-L. Florin, J. K. H. Hörber, in Methods in Molecular Biology: Kinesin Protocols, Vol. 164 (Ed.: I. Vernos), Humana Press, Totowa, 2001.

[28] E. L. Florin, A. Pralle, E. H. K. Stelzer, J. K. H. Horber, App. Phys. A 1998 66, S75.

[29] T. Wohland, A. Rosin, E. H. K. Stelzer, Optik 1996, 102, 181

[30] K. Svoboda, S.-M. Block, Cell 1994, 77, 773.

[31] M. Doi, S. F. Edwards, The Theory of Polymer Dynamics, Vol. 73, Oxfod Science Publications, 1998.

[32] N. Hirokawa, K. K. Pfister, H. Yorifuji, M. C. Wagner, S. T. Brady, G. S. Bloom, Cell 1989, 56, 867.

[33] C. Tischer, S. Altmann, S. Fisinger, J. K. H. Horber, E. H. K. Stelzer, E. L. Florin, Appl. Phys.s Lett. 2001, 79, 3878.
[34] J. Howard, A. Hunt, S. Baek, in Methods in Cell Biology, Vol 39, Vol. 39 , Academic Press Inc., San Diego, 1993, pp. 137.

[35] J. Wilhelm, E. Frey, Phys. Rev. Lett. 1996, 77, 2581.

[36] E. Frey, K. Kroy, J. Wilhelm, in Advances in Structural Biology, Vol. 5 (Eds.: S. K. Mathotra, J. A. Tutsynski), Jai Press, London, 1998.

[37] E. Frey, ChemPhysChem 2002, 3, 270.

[38] H. Grubmüller, B. Heymann, P. Tavan, Science 1996, 271, 997.

[39] E. K. O'Shea, J. D. Klemm, P. S. Kim, T. Alber, Science 1991, 254, 539.

[40] M. Eichinger, H. Heller, H. Grubmüller, in Workshop on Molecular Dynamics on Parallel Computers, John von Neumann Institute for Computing (NIC) Research Centre Jülich, Germany, 8-10 February 1999 (Eds.: R. Esser, P. Grassberger, J. Grotendorst, M. Lewerenz), World Scientific, Singapore 912805, 2000, 397.

[41] M. Eichinger, H. Grubmüller, H. Heller, P. Tavan, J. Comput. Chem. 1997, $18,1729$.

[42] F. Kozielski, S. Sack, A. Marx, M. Thormahlen, E. Schonbrunn, V. Biou, A. Thompson, E. M. Mandelkow, E. Mandelkow, Cell 1997, 91, 985.

[43] U. Henningsen, M. Schliwa, Nature 1997, 389, 93.

[44] M. Grummt, G. Woehlke, U. Henningsen, S. Fuchs, M. Schleicher, M. Schliwa, EMBO J. 1998, 17, 5536.

[45] K. Hahlen, F. Schafer, M. Schliwa, G. Woehlke, Eur. J. Cell Biol. 2003, 82, 65.

[46] W. O. Hancock, J. Howard, J. Cell Biol. 1998, 140, 1395.

[47] K. Svoboda, S. M. Block, Cell 1994, 77, 773.

[48] N. A. M. Verhaegh, A. van Blaaderen, Langmuir 1994, 10, 1427.

[49] A. Pralle, M. Prummer, E.-L. Florin, E. H. K. Stelzer, J. K. H. Hörber, Microsc. Res. Tech. 1999, 44, 378.

[50] A. Pralle, E.-L. Florin, E. H. K. Stelzer, J. K. H. Hörber, Appl. Phys. A 1998, 66, S71.

[51] M. W. Allersma, F. Gittes, M. J. deCastro, R. J. Stewart, C. F. Schmidt, Biophys. J. 1998, 74, 1074

Received: October 28, 2003

Revised: June 7, 2004 